\title{
Quantification of health effects of exposure to air pollution
}

\section{Krzyzanowski, A Cohen, R Anderson, and the WHO Working Group}

\section{Air pollution is a significant factor shaping public health}

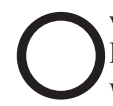
ver the past decade epidemiological studies in Europe and worldwide have measured increases in mortality and morbidity associated with air pollution. ${ }^{12}$ As evidence of health effects of air pollution has accumulated, European governments, the World Health Organisation (WHO), and other groups have begun to use data from these studies to inform environmental policies through, for example, quantitative estimates of impact of air pollution on public health. ${ }^{3-6}$ Health impact assessment involves extrapolation of exposure-health associations measured in epidemiological studies to a target population characterised by a certain observed or predicted exposure pattern. ${ }^{7}$ The analysis must address important methodological issues relevant to both its design and conduct. Clarity in defining these issues is a prerequisite for proper interpretation of the results in the policy arena. Nevertheless, the results are often prone to misinterpretation, even when the assessment is done carefully, and its multiple uncertainties are carefully presented and explained to decision makers, the press, and the public.

WHO recently convened a Working Group to examine key methodological issues in health impact assessment of air pollution using epidemiological data, and to recommend approaches to the conduct of such assessments. The Working Group comprised experts who develop and apply methods for health risk analysis, and scientists involved in the communication of the results of the analysis to the public or decision makers. The Working Group formulated recommendations on the framework and methods of health impact assessment. Their discussions focused on the conditions pertinent to the developed countries, where most of the studies documenting the relations between air pollution and health were performed. The WHO report presenting the Working Group discussions and its recommendations has been published recently. ${ }^{8}$ This paper summarises the WHO report and communicates its main messages to a broader group of public health and environmental management professionals interested in health impact assessment of air pollution.

\section{FRAMEWORK OF HEALTH IMPACT ASSESSMENT OF AIR POLLUTION}

The basic steps in health impact assessment include selection of the (set of) health outcomes associated with air pollution, adoption of the risk estimates (exposure-response function) based on epidemiological studies, and application of this function to the distribution of exposure experienced by the target population. ${ }^{7}$ The result of the analysis is the quantification of the expected health burden owing to the exposure in the target population, expressed in terms of the number of cases or years of life lost attributable to the exposure. This analysis can be extended to include economic valuation of the impact, ${ }^{6}$ but this aspect was not a subject of the Working Group discussion. Assumptions and choices made at each step may influence the result of the analysis. The WHO group discussed merits and limitations of various sources on information necessary for health impact assessment, and recommended the choices suggested by the present understanding of the health aspects of air pollution.

\section{CHOICE OF HEALTH OUTCOMES REPRESENTING THE IMPACT OF AIR POLLUTION}

Exposure to outdoor air pollution is associated with a broad spectrum of acute and chronic health effects ranging from irritant effects to death. ${ }^{9}{ }^{10}$ This suggests that the total impact is likely to exceed that contributed by the less frequent, more severe outcomes, and, in some cases, may be dominated by the less severe, but more frequent, ones. The choice of health outcome will ultimately depend on the objective of the health impact assessment. In part, it will also depend on the usefulness of the impact indices in the subsequent valuation analyses.

Epidemiology has provided much of the existing evidence for these associations, and toxicology and clinical sciences have provided convincing support for the mechanisms of many (though not all) of the observed associations. The most relevant information on the relation between mortality and air pollution provides two epidemiological designs: time series studies of daily mortality, and cohort studies of mortality over extended periods. Time series studies of mortality and air pollution use relatively widely available data on daily counts of death in a city and daily air pollution levels. They have been completed in more than 160 cities worldwide and provide solid evidence on the adverse effects of air pollution on health. However, because chronic effects of long term exposure cannot be fully quantified in such studies, some deaths attributable to air pollution will be missed and the extent to which air pollution advances the time of death cannot be quantified. ${ }^{11}{ }^{12}$

Another source of information on the impact of air pollution on mortality is cohort studies, in which large populations are followed for years and their mortality ascertained. The number of such studies is still very limited: currently, only three US studies ${ }^{13-16}$ provide such estimates; they have been extensively used for impact assessment. Several cohort studies (for example, Hoek and colleagues ${ }^{17}$ ) have been undertaken in Europe and their results should be available in the next few years.

The Working Group considered the relative contributions to health impact assessment of the two designs and concluded that both could contribute useful, albeit different, information. The following recommendations were formulated:

- The most complete estimates of both attributable numbers of deaths and average reductions in lifespan associated with exposure to air pollution are those based on cohort studies. Until the risk estimates from the European studies are available, impact assessment will need to rely on the results of currently available US studies. Additional cohort studies, in Europe and elsewhere, and confirmation of the transferability of US results to European populations are critical research needs.

- Time series studies of daily mortality, which are likely to provide a lower bound on the number of attributable deaths, and which can be conducted relatively easily in diverse locations, will continue to be valuable for: demonstrating and documenting the adverse effects of air pollution in specific locales; quantifying the effects of short term variation of air pollution (including air pollution episodes); and serving as the basis for air pollution alert systems. They will also likely continue to contribute to scientific understanding by identifying factors that modify the effects of air pollution on mortality and morbidity, toxic 
components of the air pollution mixture, and high risk subgroups, and by furthering understanding of exposure-response relations.

In some cases, increased risk of mortality estimated in time series studies may be connected with substantial loss of life years and may be directly used for quantification of air pollution impact. This might occur, for example, when air pollution increases the risk of mortality from acute respiratory infections in children, as shown in several studies in developing countries.

Considering the wide range of indicators of morbidity associated with air pollution, the Working Group suggested that impact measures which integrate various clinical manifestations of a disease, and provide estimates of the effects on quality of life, are to be preferred. Such measures focus on the end consequences of pollution related illness rather than on the pathological or clinical aspects. Restricted activity days, which include operational concepts such as missed work or school days, as well as reduced physical activities, are concrete, quantifiable, and easily communicated. However, more research is needed to quantify the relation of these measures with air pollution exposure, as there have been few studies using this type of outcome.

\section{WHICH COMPONENTS OF RISK ESTIMATES MADE IN ONE POPULATION CAN BE TRANSFERRED (GENERALISED) TO ANOTHER?}

Health impact assessments usually apply air pollution effect estimates derived from a study in one population (the evidentiary population), to estimate impacts in another (the target population). Such assessments assume that the effect estimates in the evidentiary population are transferable, or generalisable, to the target population. The validity of this assumption implicitly requires that the two populations be similar with regard to factors that influence the magnitude of the effect estimates, such as structure of the morbidity, basic health status, or composition of the air pollution mix. Recent analyses have begun to explore how such factors may explain the variation in air pollution effect estimates observed among locations in Europe and the United States. ${ }^{218-20}$ They suggest the presence of significant and real heterogeneity in location specific estimates that may need to be taken into account in health impact assessments. However, at present knowledge about effect modifiers is quite limited. Until we have a more complete understanding of these factors, their value for health impact assessment will also be limited.
In general, the most precise valid effect estimate should be used for impact assessment. In some cases, that may be the estimate from the target population itself. However, in many cases the local estimate, if it exists, may not be the most precise (or valid) estimate, because of random error or epidemiological bias. Therefore, health impact assessments in specific locales should consider using risk estimates from multisite studies or meta-analytic summary estimates in the absence of compelling evidence that the target population differs from the aggregate vis-à-vis its response to air pollution.

The transferability of the mortality effect estimates from the US cohort studies to other, non-US, target populations can be justified on the basis that: (1) these estimates are the only ones that currently exist; (2) they are the only ones which are theoretically justifiable. Ideally, application of these estimates to other target populations should incorporate information on factors that influence the magnitude of the mortality coefficients and cause them to differ among populations. Unfortunately, lack of knowledge precludes this at present.

Transferability of baseline mortality and morbidity rates among European populations cannot be implicitly assumed for purposes of impact assessment. With regard to mortality, population specific rates, compiled using relatively standardised approaches, are widely available. They should, of course, be used in this context. Differences in recording and classification of cause specific morbidity among countries lead to non-comparability of baseline rates. Better data on the baseline rates of key morbidity outcomes is a priority for strengthening the capability to perform health impact assessments.

Considering the above issues, the Working Group formulated the following recommendation: "Health impact assessments should exercise great care when the evidentiary and target populations differ. In general, the most precise, valid and specific effect estimate should be used for impact assessment. The deviations of the conditions in the target population from those in the evidentiary population which would plausibly affect estimated pollution effects must be made explicit and, whenever possible, should be included in the uncertainty analysis."

\section{HOW SHOULD EXPOSURE TO AIR POLLUTION BE CHARACTERISED FOR THE PURPOSE OF A HEALTH IMPACT ASSESSMENT?}

Recent analyses suggest that there is no discernable threshold for the effects of particulate air pollution on daily or longer term average mortality from cardiorespiratory disease. ${ }^{18}{ }^{21}$ though for other pollutants, such as ozone, the evidence is not as clear. Although this provides some theoretical justification for calculating impacts based on exposure levels down to and even including so-called "background" (possibly nonanthropogenic) levels, the Working Group recommended that in most cases impacts should be calculated for a range of population exposure levels that reflect realistic policy options. Depending on the pollutant, those options might include an ambient concentration of zero, some non-zero "clean" concentration, or a concentration mandated by an air quality standard. Since the estimates of impact may be sensitive to the chosen range of exposure, ${ }^{3}$ the chosen range should be clearly defined and the effect of that choice on the impact estimates should be included in the sensitivity analysis.

The Working Group concluded that health impact assessments should design exposure characterisation in the target population to mirror as nearly as possible exposure in the study providing the effect estimate. Impact assessments should avoid adding estimates of effects of individual pollutants derived from single pollutant statistical models unless there is a good reason to assume that various pollutants from the air pollution mixture affect health independently.

\section{HOW TO ASSESS AND REDUCE UNCERTAINTY OF IMPACT ASSESSMENT?}

Health impact assessments should address the uncertainties in their estimates of impact in as explicit and quantitative a manner as possible. They should indicate how deviations from key assumptions would be expected to affect the results of the assessment and their application in policy analyses. The specific content of the uncertainty analysis will, therefore, depend on its purpose (for example, in consideration of various policy options, or in scientific investigation). The uncertainties in such assessments include those of the effect estimates (random error, bias, and confounding), as well as those associated with generalising those estimates to target populations. Therefore, the standard measures of statistical precision of epidemiological estimates ( $p$ values, confidence intervals) alone are not sufficient. The magnitude of the uncertainty should be tested by sensitivity analysis. It should focus on the assumptions and input parameters which are the most important determinants of the magnitude of the estimated impacts.

Research to quantify chronic effects of pollution, to identify the determinants of variation in health response to an exposure between various populations, as 
well as to quantify the impacts of air pollution on disease burden, are the most needed to improve the scope and reliability of health impact analysis, as well as to reduce its uncertainty. The research should be specific to target populations and provide support for generalisation of the studies to wider target populations. The currently implemented WHO project to assess the burden of air pollution to health in all parts of the world (Comparative Risk Assessment project) emphasises the need for the evidence necessary to expand such assessments to the developing countries. In those parts of the world, exposure situation, population health status and, possibly, susceptibility to air pollution are different from those in the developed parts of the world. The few available studies confirm, however, that the daily changes in air pollution are associated with changes in mortality in a similar way to the one observed in the developed countries. This suggests that air pollution is a significant factor shaping public health in the developing countries.

Occup Environ Med 2002;59:791-793

\section{Authors' affiliations}

M Krzyzanowski, WHO European Centre for Environment and Health, Bonn Office, Germany A Cohen, Health Effects Institute, Boston,

Massachusetts, USA

R Anderson, St George's Hospital Medical

School, London, UK

Working Group members: M Amann,

$R$ Anderson, J Ayres, T Bellander, L van Bree,

B Brunekreef (Chairman), A Cohen (Rapporteur)

J Dowie, N Englert, F Forastiere, I Hertz-Piciotto,

$G$ Hoek, F Hurley, K Katsouyanni, M

Krzyzanowski (Sc. Secretary), N Kuenzli,

A Le Tertre, DJ Maddison, M Martuzzi,

R Maynard, B Miller, B Ostro, A Pruess, R Torfs;

Invited reviewers: R Burnett, AEM de Hollander,

T Louis, J Pekkanen, EH Wichmann
Correspondence to: Dr M Krzyzanowski, WHO European Centre for Environment and Health, Bonn Office, Goerrestrasse 15, 53113 Bonn, Germany; mkr@ecehbonn.euro.who.int

Accepted 9 April 2002

\section{REFERENCES}

1 WHO. Air Quality Guidelines for Europe, 2nd edn. WHO Regional Publications, European Series, No. 91. Copenhagen: WHO Regional Office for Europe, 2000.

2 Health Effects Institute. National morbidity, mortality and air pollution study. HEl Report 94, Part 2, 2000

3 Künzli N, Kaiser R, Medina S, et al. Public-health impact of outdoor and traffic-related air pollution: a European assessment. Lancet 2000;356:795-801

4 Bellander T, Svartengren M, Berglind N, et al. The Stockholm Study on Health Effects of Air Pollution and their Economic Consequences. Part II: Particulate matter, nitrogen dioxide, and health effects. Exposure-response relations and health consequences in Stockholm County (SHAPE). Department of Environmental Health Karolinska Hospital. Publikation 1999: 160. Vägverket, Butiken, Stockholm, December 1999.

5 Hurley F, Holland MR, Markandya A, et al. Towards assessing and costing the health impacts of ambient particulate air pollution in the UK. Institute of Occupational Medicine Report TM/00/07. Edinburgh, December 2000

6 Department of Health, Ad-Hoc Group on the Economic Appraisal of the Health Effects of Air Pollution. Economic appraisal of the health effects of air pollution. London: The Stationery Office, 1999.

7 WHO. Evaluation and use of epidemiological evidence for environmental health risk assessment. Copenhagen, WHO Regional Office for Europe, 2000, EUR/00/5020369 (E68940) (also: Environmental Health Perspectives 2000;108:997-1002).

8 WHO. Quantification of health effects of exposure to air pollution. Report on a WHO Working Group. Bilthoven, Netherlands, 20-22 November 2000. WHO Regional Office for Europe, 2001, EUR/01/5026342 (E74256)

9 ATS, Committee of the Environmental and Occupational Health Assembly of the American Thoratic Society. Health effects of outdoor air pollution, Part 1. Am J Respir Crit Care Med 1996:153:3-50.
10 ATS, Committee of the Environmental and Occupational Health Assembly of the American Thoratic Society. Health effects of outdoor air pollution, Part 2. Am J Respir Crit Care Med 1996;153:477-98.

11 McMichael AJ, Anderson HR, Brunekreef B, et al. Inappropriate use of daily mortality analyses to estimate longer-term mortality effects of air pollution. Int J Epidemio 1998;27:450-3.

12 Künzli N, Medina S, Kaiser R, et al. Assessment of deaths attributable to air pollution: should we use risk estimates based on time series or cohort studies? Am J Epidemiol 2001;153:1050-5

13 Dockery DW, Pope A 3rd, Xu X, et al. An association between air pollution and mortality in six United States cities. N Engl J Med 1993;329:1753-9.

14 Pope CA III, Thun M, Namboodiri M, et al. Particulate air pollution as a predictor of mortality in a prospective study of United States adults. Am J Respir Crit Care Med 1995: 151:669-74.

15 Abbey DE, Nishino N, McDonnell WF, et al. Long-term inhalable particles and other air pollutants related to mortality in nonsmokers. Am J Respir Crit Care Med 1999:159:373-82.

16 Pope CA III, Burnett R, Thun $M$, et al. Lung cancer, cardiopulmonary mortality, and long-term exposure to fine particulate air pollution. JAMA 2002;287: 1 132-41.

17 Hoek G, Fischer P, Van Den Brandt P, et al Estimation of long-term average exposure to outdoor air pollution for a cohort study on mortality. J Expo Anal Environ Epidemiol 2001;11:459-69.

18 Health Effects Institute. Special Report: Reanalysis of the Harvard Six Cites Study and the American Cancer Society Study of Particulate Air Pollution and Mortality. HEI, July 2000.

19 Katsouyanni K, Touloumi G, Samoli E, et al. Confounding and effect modification in the short-term effects of ambient particles on total mortality: results from 29 European cities within the APHEA 2 project. Epidemiology 2001;12:521-31.

20 Levy JI, Hammitt JK, Spengler JD, et al. Estimating the mortality impacts of particulate matter: what can be learned from between-study variability? Environ Health Perspect 2000;108:109-17.

21 Daniels MJ, Dominici F, Samet J, et al. Estimating particulate matter-mortality dose-response curves and threshold levels: an analysis of daily time-series for the 20 largest United States cities. Am J Epidemiol 2000;152:397-406

(1) (a) true; (b) true; (c) true; (d) true; (e) false

(2) (a) true; (b) true; (c) true; (d) false; (e) true

(3) (a) true; (b) false; (c) true; (d) false; (e) false

(4) (a) true; (b) false; (c) false (d) false; (e) true 